\title{
A CONTINUITY PROPERTY WITH APPLICATIONS TO THE TOPOLOGY OF 2-MANIFOLDS( $\left.{ }^{1}\right)$
}

\author{
BY \\ NEAL R. WAGNER
}

\begin{abstract}
A continuity property is proved for variable simply connected domains with locally connected boundaries. This theorem provides a link between limits of conformal mappings and of retractions. Applications are given to the space of retractions of a compact 2-manifold $M^{2}$, where it is shown that the space of deformations retractions is contractible and the space of nullhomotopic retractions has the same homotopy type as $M^{2}$. Other applications include a proof that the space of retracts of $M^{2}$ (with a natural quotient topology) is an absolute neighborhood retract, and a type of global solution to the Dirichlet problem.
\end{abstract}

0 . Introduction. This article contains proofs for the results announced in [23]. The basic tool for this work is a continuity property for variable simply connected domains with locally connected boundaries (Theorem 2.2 ), which provides a link between limits of conformal mappings and of retractions. Preliminary material on conformal mapping theory appears in $\$ 1$, including a continuity property for a fixed domain (Theorem 1.1) similar to Theorem 2.2.

In $\S \S 3$ and 4, we use Theorem 2.2 to analyze two components of the space of retractions of an arbitrary compact 2-manifold $M^{2}$. In $\S 3$, we construct a contraction of the space of deformation retractions of $M^{2}$, while in $\S 4$ we show that the space of nullhomotopic retractions of $M^{2}$ has the same homotopy type as $M^{2}$. The methods of $\S 4$ are similar to those in [21] and [22]. In both $\S \S 3$ and 4 , the construction is essentially that first given by Alexander [2], [11, p. 524], as modified for retractions by Borsuk [4] and the author (Remark 3.2). Only in five cases (the 2-sphere, disk, annulus, projective

Received by the editors June 28, 1973 and, in revised form, December 10, 1973.

AMS (MOS) subject classifications (1970). Primary 30A30, 54C15, 57A05; Secondary 54C35, 54C55, 54F40, 55D10, 55F05.

Key words and phrases. Absolute neighborhood retract, Carathéodory convergence, compact-open topology, conformal mapping, continuity property, cross section, Dirichlet problem, Fréchet convergence, homotopy equivalence, locally trivial fibre space, prime end of the first kind, retract, retraction, Schoenflies theorem, two-manifold, variable domain.

(1) The final stage of this work was supported by a grant from the University Research Institute of the University of Texas at El Paso. 
plane and Möbius strip) does this work provide a complete analysis of the global properties of the space of retractions. For example, even in cases as simple as the torus or the disk with three holes, the space of retractions has a complicated infinite array of components [24].

In $\S 5$, we give the set of retracts of $M^{2}$ the quotient topology determined by the projection from the space of retractions, and prove that the space of retracts is an absolute neighborhood retract (Theorem 5.5). We also give a version for retracts of the continuity property (Theorem 5.4).

Finally, in $\S 6$ we present two global forms of the continuity property: a canonical form of the Schoenflies theorem and a type of simultaneous solution to the Dirichlet problem.

Part of the material in $\S 3$ appeared in the author's doctoral dissertation at the University of Illinois, Urbana-Champaign. The author would like to thank his advisor, Mary-Elizabeth Hamstrom, and the referee of [21] for a number of very useful ideas. He would also like to thank C. W. Neville and J. M. Gray for encouragement.

1. Preliminaries on conformal mapping. In this section we introduce concepts from conformal mapping theory which will be used in §2. As basic references for this section, see [1], [10], and [15]. Throughout this paper, $B^{2}$ will denote the closed unit ball in the Euclidean plane $E^{2}$, and $C_{\alpha}$ will denote the circle with center at the origin and radius $\alpha$. The symbol $A(J, K)$ denotes the closed annular region bounded by simple closed curves $J$ and $K$, where $J$ lies in the bounded complementary domain of $K$. In particular, $A^{2}$ is used for $A\left(C_{1}, C_{2}\right)$. The abbreviations bdry, cl, int, im, diam, and dist are used respectively, for boundary, closure, interior, image, diameter, and distance.

Let $G$ be a bounded simply connected domain in $E^{2}$. A point $v \in F=$ $\operatorname{bdry}(G)$ is accessible from $G$ if there is an embedding $e:[0,1] \rightarrow G \cup F$ such that $e([0,1)) \subset G$ and $e(1)=v$. An accessible point of $F$ consists of a point $v \in F$ and an equivalence class of embeddings, where we take $e_{1}$ equivalent to $e_{2}$ if and only if there is an embedding $e$ as above which has image points in common with $e_{1}$ and $e_{2}$ arbitrarily close to $v$. By an abuse of language, we shall refer to $v$ or to $(v, e)$ as an accessible point.

Given distinct accessible points $\left(v_{1}, e_{1}\right)$ and $\left(v_{2}, e_{2}\right)$ of $F$, it is easy to find a mapping $E:[-1,1] . G \cup F$ such that

$$
E((-1,1)) \subset G, \quad E(-1)=v_{1}, \quad E(1)=v_{2},
$$

and, for some $\epsilon>0$,

$$
E(-t)=e_{1}(t) \text { and } E(t)=e_{2}(t)
$$

for all $t \in[1-\epsilon, 1]$. The set $E([-1,1])$ is salled a cross cut and splits $G$ 
into two components. A closed $H$-interval of $F$ is the collection of accessible points of $F$ which are accessible from one of the above two components [15, p. 59]. Thus $E$ splits the accessible points of $F$ into two $H$-intervals with only the endpoints $\left(v_{1}, e_{1}\right)$ and $\left(v_{2}, e_{2}\right)$ in common.

A decreasing sequence $\left\{H_{n}\right\}$ of $H$-intervals containing at most one accessible point is said to determine a prime end of $G$. There is a corresponding decreasing sequence $\left\{G_{n}\right\}$ of subdomains of $G$ such that the accessible points of $F$ accessible from $G_{n}$ are exactly those in $H_{n}$ and such that $P=\cap \operatorname{cl}\left(G_{n}\right)$ is contained in $F$. The set $P$ depends only on the prime end determined by $\left\{H_{n}\right\}$, and we say that $\boldsymbol{P}$ belongs to this prime end. If the set which belongs to a prime end consists of one accessible point, we say the prime end is of the first kind.

The following known theorem is reminiscent of the result in [18, p. 262], where conditions are given equivalent to the existence of a conformal homeomorphism $f: \operatorname{int}\left(B^{2}\right) \rightarrow G$.

\subsection{THEOREM (CONTINUITY PROPERTY FOR A FIXED DOMAIN). Let $\boldsymbol{G}$} be a bounded simply connected domain with boundary $F$, and let $D$ be a closed disk contained in $G$. The following are equivalent.

(a) The boundary $F$ is locally connected.

(b) The prime ends of $G$ are all of the first kind.

(c) There is a continuous surjection $f: B^{2} \rightarrow G \cup F$ which is a conformal homeomorphism on $\operatorname{int}\left(B^{2}\right)$.

(d) There is a retraction $\varphi$ of $E^{2} \operatorname{lint}(D)$ with image $E^{2} \backslash G$.

(e) There is a canonical retraction $\psi$ of $E^{2} \backslash \operatorname{int}(D)$ with image $E^{2} \backslash G$ mapping $G \backslash \operatorname{int}(D)$ onto $F$.

1.2. Remark. Compare this theorem with Theorems 2.2 and 5.4. See $[26$, p. 112] for three other topological conditions (one appearing in the proof below) equivalent to (a) through (e) above. The equivalence of (b) and (c) is an old result due to Carathéodory and others. A direct proof that (a) implies (d) was originally given by Borsuk [3]. In condition (e) above and in Theorem 2.2 (e), the word "canonical" means that, for any boundary $F$ and any disk $D$ in $G$, we are giving a procedure for constructing a unique retraction $\psi$ with the desired properties.

Proof. To prove that (a) implies (b), assume $F$ is locally connected. Whyburn proves [26, p. 112] this is equivalent to saying each $v \in F$ is accessible from all sides from $G$, meaning that given any cross cut in $G, v$ is accessible from either (or both) of the resulting components which has $v$ on its boundary. It is then easy to see that (b) is true $[15$, p. 65]. The proof that (b) implies (c) can be found in $[15$, p. 67]. 

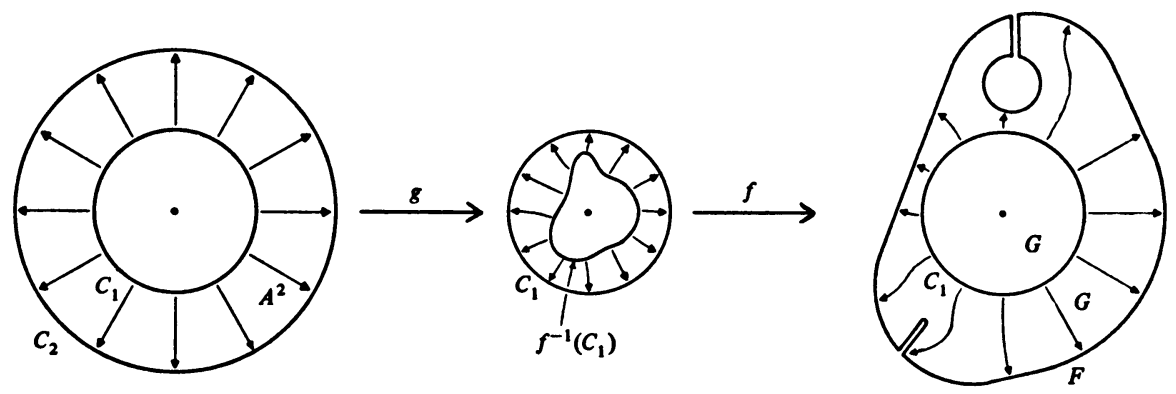

\section{Figure 1}

Next, we give a constructive proof that (c) implies (e). Without loss of generality we can assume that the closed disk $D$ is the closed unit disk $B^{2}$. Let $g: A^{2}=A\left(C_{1}, C_{2}\right) \rightarrow A\left(f^{-1}\left(C_{1}\right), C_{1}\right)$ be a homeomorphism which is a radial contraction of a map conformal on the interior of the annulus. (See Figure 1.) Assuming we specify that $f$ fixes the origin, the maps $f$ and $g$ and hence $h=f \circ g$ are only determined up to a rotation, but the construction which follows is independent of rotations. Let $\lambda: A^{2} \rightarrow C_{2}$ be the obvious radial retraction. We define a retraction $\psi$ of $E^{2} \backslash \operatorname{int}\left(B^{2}\right)$ onto $E^{2} \backslash G$ by setting $\psi$ equal to $h \circ \lambda \circ h^{-1}$ on $(G \cup F) \backslash \operatorname{int}\left(B^{2}\right)$ and $\psi$ equal to the identity on $E^{2} \backslash G$. The continuity of $\psi$ only needs checking on $F$, and it is continuous there because $h$ is continuous on $C_{2}$.

It is trivial that (e) implies (d), and assuming (d), a bounded portion of $E^{2} \backslash G$ is a locally connected continuum [26, p. 26], and so its boundary $F$ is also locally connected [26, p. 106]. Q.E.D.

For each nonnegative integer $n$, suppose there is given a bounded simply connected domain $G_{n}$ in the plane such that some fixed closed disk $D$ with a point $u_{0}$ as center is contained in all the $G_{n}$. Let $f_{n}: \operatorname{int}\left(B^{2}\right) \rightarrow G_{n}$ be the unique conformal homeomorphism mapping the origin to $u_{0}$ and having positive derivative at the origin $[15$, p. 13$]$.

1.3. Definition. The domain $G_{0}$ is the kernel (relative to $u_{0}$ ) of the sequence $\left\{G_{n}: n \geqslant 1\right\}$ if $G_{0}$ is the largest domain containing $u_{0}$ such that each compact subset of $G_{0}$ is contained in $G_{n}$ for sufficiently large $n$. (Alternatively, we can let $G$ be the set of points with a neighborhood contained in the $G_{n}$ for sufficiently large $n$. The component of $G$ containing $u_{0}$ is the kernel.) The sequence $\left\{G_{n}\right\}$ Carathéodory converges (relative to $u_{0}$ ) to $G_{0}$ if every subsequence of $\left\{G_{n}\right\}$ has kernel. $G_{0}$.

1.4. Theorem (CARAthéodory). The sequence $\left\{G_{n}\right\}$ Carathéodory converges to $G_{0}$ if and only if the sequence $\left\{f_{n}\right\}$ converges to $f_{0}$ uniformly on compact subsets of int $\left(B^{2}\right)$. 
The same theorem is true for the functions $f_{n}^{-1}$. (See [15] for proofs and [8] for references.)

We shall also need versions of Theorems 1.1 and 1.4 for a general (second countable) 2-manifold $M^{2}$. Our technique will be to lift the simply connected domain $G$ to the universal covering space of $M^{2} \backslash \partial M^{2}$, which will be $E^{2}$ except for the cases where $M^{2}$ is the projective plane or the 2-sphere [1, p. 104]. Since $G$ is simply connected and hence contractible in $M^{2}$, it is not hard to see that $G$ will lift to homeomorphic copies in any covering space [19, pp. 66-68]. We shall start with a precompact simply connected domain $G$ in $M^{2}$, and since we wish to obtain a bounded simply connected domain in $E^{2}$, we employ the following strategem: Attach a collar $\partial M^{2} \times[0,1]$ to $\partial M^{2}$, producing a 2-manifold $N^{2}$, and take the universal covering space of $N^{2} \backslash \partial N^{2}$. If we suppose that $M^{2}$ has been given a conformal structure so as to make it a

Riemann surface (a bordered Riemann surface if $\partial M^{2} \neq \varnothing$ and including conjugates of conformal mappings if $M^{2}$ is not orientable [1]), then we can extend this to a conformal structure for $N^{2}$ as in [1, p. 118]. Finally, we can put a conformal structure on the universal covering space so as to make the covering projection conformal [1, p. 119].

If $F$, the boundary of $G$, consists of more than one point, then $G$ is of hyperbolic type $[1$, pp. $141,158,204]$, i.e., conformally equivalent to $\operatorname{int}\left(B^{2}\right)$. Let $\hat{G} \subset E^{2}$ be a component of $p^{-1}(G)$, where $p: E^{2} \rightarrow N^{2} \backslash \partial N^{2}$ is the covering projection. While $\hat{F}=\operatorname{bdry}(\hat{G})$ need not be homeomorphic to $F, \hat{F}$ will be locally connected if $F$ is. Thus by Theorem $1.1(\mathrm{c})$, there is a continuous function $\hat{f}: B^{2} \rightarrow \hat{G} \cup \hat{F}$ which is a conformal homeomorphism on $\operatorname{int}\left(B^{2}\right)$. The composition $f=p \circ \hat{f}$ gives a continuous surjection of $B^{2}$ onto $G \cup F$ which is conformal on $\operatorname{int}\left(B^{2}\right)$. Hence we have the following.

\subsection{THEOREM. In an arbitrary (second countable) 2-manifold $M^{2}$, let $G$} be a precompact simply connected domain with boundary $F$ consisting of more than one point. Let $D$ be a closed disk in $G$ containing some $u_{0} \in M^{2}$ in its interior. Then the five conditions of Theorem 1.1 make sense and are equivalent in this more general setting.

For each nonnegative integer $n$, suppose $G_{n}$ is a precompact simply connected domain in $M^{2}$ of hyperbolic type such that each $G_{n}$ contains some fixed closed disk $D$ with $u_{0}$ in its interior. Definition 1.3 may be used in the same form to define Caratheodory convergence of $\left\{G_{n}\right\}$ to $G_{0}$. For each $n$ let $f_{n}$ be a conformal homeomorphism of $\operatorname{int}\left(B^{2}\right)$ onto $G_{n}$ mapping the origin to $u_{0}$. Each $f_{n}$ is uniquely determined up to a rotation, and a reflection if $M^{2}$ is not orientable. Thus we require that the images of the positive $x$-axis 
under the $f_{n}$ all make the same angle at $u_{0}$, and, if $M^{2}$ is not orientable, we require that each $f_{n}$ be directly conformal using a fixed orientation for $D$ and $\operatorname{int}\left(B^{2}\right)$.

1.6. THEOREM. Using notation above, $\left\{G_{n}\right\}$ Carathéodory converges to $G_{0}$ if and only if $\left\{f_{n}\right\}$ converges to $f_{0}$ uniformly on compact subsets of $\operatorname{int}\left(B^{2}\right)$.

Finally, we state a lemma which is very useful in work with variable domains.

1.7. Lemma (LindelöF [10, p. 33]). Suppose an analytic function $f$ is bounded on some bounded domain $G$ (containing a point $u_{0}$ ) by a number $M>0$, and suppose that for some $r>0$, there is an arc of the circle $C=$ $\left\{u:\left|u-u_{0}\right|=r\right\}$ disjoint from $\operatorname{cl}(G)$, where the arc makes an angle of $2 \pi / k$ for some integer $k$. Suppose, further, there is a number $m>0$ such that for any boundary point $w$ of $G$ inside $C$, we have $\limsup _{u \rightarrow w}|f(u)| \leqslant m$. Then $\left|f\left(u_{0}\right)\right| \leqslant\left(m M^{k-1}\right)^{1 / k}$.

2. The continuity property for variable domains. In this section we give an altered version of a theorem of D. Gaier [8, p. 395]. We have added three new equivalent conditions, but weakened the generality of the theorem. For other references, see [8] and [9, p. 27].

For each nonnegative integer $n$, let $G_{n}$ be a bounded simply connected domain in $E^{2}$ such that each $G_{n}$ has locally connected boundary $F_{n}$ and contains some fixed closed disk $D$ with a point $u_{0}$ as center. Let $f_{n}: B^{2} \rightarrow$ $G_{n} \cup F_{n}$ be the function given by Theorem 1.1(c), which will be uniquely determined by requiring that $f_{n}$ map the origin to $u_{0}$ and that $f_{n}$ have positive derivative at the origin. Following $[25$, p. 337] and [8, p. 394], we give a definition which introduces a measure of the "smoothness" of the boundary $F_{n}$ of our domain $G_{n}$.

2.1. Definition. For every $\delta>0$, consider all cross cuts $Q$ in $G_{n}$ missing $u_{0}$ and of diameter $\operatorname{diam}(Q) \leqslant \delta$, and let $d\left(G_{n} \backslash Q\right)$ denote the diameter of the component of $G_{n} \backslash Q$ which does not contain $u_{0}$. Set

$$
\eta_{n}(\delta)=\sup \left\{d\left(G_{n} \backslash Q\right): \operatorname{diam}(Q) \leqslant \delta\right\} .
$$

One says that the sequence $\left\{F_{n}: n \geqslant 1\right\}$ Fréchet converges to $F_{0}$ if

(1) $\left\{G_{n}\right\}$ Carathéodory converges to $G_{0}$ (Definition 1.3), and

(2) $\lim _{n \rightarrow \infty, \delta \rightarrow 0} \eta_{n}(\delta)=0$.

2.2. Theorem (Continuity PROPERTY For VARIAble Domains). The following conditions are equivalent. 
(a) The sequence $\left\{F_{n}\right\}$ Fréchet converges to $F_{0}$.

(b) Each accessible point $v_{0} \in F_{0}$ is the limit of a sequence $\left\{v_{n} \in F_{n}\right\}$ of accessible points, and every subsequence of such a sequence has in turn a convergent subsequence. (See Remark 2.3(1) below.)

(c) The sequence $\left\{f_{n}\right\}$ converges to $f_{0}$ uniformly on the closed disk $B^{2}$.

(d) There are retractions $\varphi_{n}(n \geqslant 0)$ of $E^{2} \backslash \operatorname{int}(D)$ with image $E^{2} \backslash G_{n}$ such that $\left\{\varphi_{n}\right\}$ converges to $\varphi_{0}$ uniformly on $E^{2} \backslash \operatorname{int}(D)$.

(e) There are canonical retractions $\psi_{n}(n \geqslant 0)$ satisfying (d) such that each $\psi_{n}$ maps $G_{n} \backslash \operatorname{int}(D)$ onto $F_{n}$.

2.3. REMARK. (1) Since each $F_{n}$ is locally connected, all prime ends are of the first kind, and the notions of "prime end" and "accessible point" coincide (see Theorem 1.1). We say that a sequence $\left\{v_{n} \in F_{n}\right\}$ of accessible points converges to an accessible point $v_{0} \in F_{0}$ if there are representing embeddings $e_{n}$ for $v_{n}(n \geqslant 0)$ such that $\left\{e_{n}\right\}$ converges to $e_{0}$ uniformly on $[0,1]$.

(2) It is not hard to see that condition (b) makes sense and is equivalent to (a) even in the more general case considered in $\left[8\right.$, p. 395], where only $F_{0}$ is assumed locally connected and the convergence is assumed uniform only on $\operatorname{int}\left(B^{2}\right)$. We choose to include condition (b) here because it may be of interest in its own right. For the proof of Theorem 2.2, we could partly refer to [8], but instead we shall outline an independent proof, relying on Lindelöf's lemma (Lemma 1.7) rather than the Dirichlet integral that is used in [8].

(3) We could increase the similarity between Theorems 1.1 and 2.2 by changing condition (a) of Theorem 1.1 to $\lim _{\delta \rightarrow 0} \eta_{0}(\delta)=0$, which is clearly equivalent to the local connectedness of $F_{0}[8$, p. 394]. Similarly, condition (b) of Theorem 1.1 could also be altered.

Proof that (a) implies (b). Assume (a) is true and (b) is not true. The Caratheodory convergence implies the first condition of (b), so we can assume there is a sequence $\left\{v_{n} \in F_{n}: n \geqslant 1\right\}$ of prime ends with no convergent subsequence. Using the map $f_{0}$, we define standard simple closed curves $J(\alpha)$ and standard radial arcs $A(\gamma)$ in $G_{0}$ to be images under $f_{0}$ of circles $C_{\alpha}(\alpha<1)$, and of rays from the origin making an angle $\gamma$, respectively.

By taking a subsequence of $\left\{v_{n}\right\}$ (but keeping the same notation), we claim that we can choose a sequence $0<\alpha_{1}<\alpha_{2}<\alpha_{3}<\cdots$ converging to 1 and an $\epsilon>0$ such that

(1) for all $n$, each point of $J\left(\alpha_{n}\right)$ can be joined to the corresponding point of $F_{0}$ by a portion of a standard arc of diameter less than $1 / n$ lying except for its endpoints in $G_{0}$ but outside $J\left(\alpha_{n}\right)$,

(2) for all $n, J\left(\alpha_{n}\right)$ is a subset of $G_{n}$,

(3) for all $n$, each point of $F_{n}$ is within $\beta_{n}$ of $F_{0}$, where $\beta_{n}=$ 
$\operatorname{dist}\left(J\left(\alpha_{n}\right), F_{0}\right)$ (note that $\beta_{n}<1 / n$ ), and

(4) for all representing embeddings $e_{n}$ of $v_{n}$ such that $e_{n}(0)=u_{0}$, there is a terminal arc of $e_{n}([0,1])$ outside $J\left(\alpha_{n}\right)$ having diameter greater than or equal to $\epsilon$.

In condition (1) we use the fact that $F_{0}$ is locally connected, and in conditions (2) and (3) we take subsequences. If (4) were not true, we could construct a convergent subsequence of $\left\{v_{n}\right\}$ using the standard arcs $A(\gamma)$.

For the rest of this proof, assume that $n$ is fixed and assume that $e_{n}$ is a fixed representing embedding for $v_{n}$. For each angle $\gamma$, we use $A(\gamma)$ and $e_{n}$ to attempt to construct a representing embedding $e_{\gamma}$ for $v_{n}$ as follows:

(5) Trace along $A(\gamma)$ to $J\left(\alpha_{n}\right)$ (using parameter values in $\left[0,1-\alpha_{n}\right]$ and the map $\left.f_{0}\right)$. Then, if possible, connect up with that point of $e_{n}([0,1])$ outside $J\left(\alpha_{n}\right)$ with largest parameter value (denoted $t_{\gamma}$ ) using an arc of diameter less than or equal to $2 / n$. Finally, finish the embedding along $e_{n}([0,1])$. (See Figure 2.)

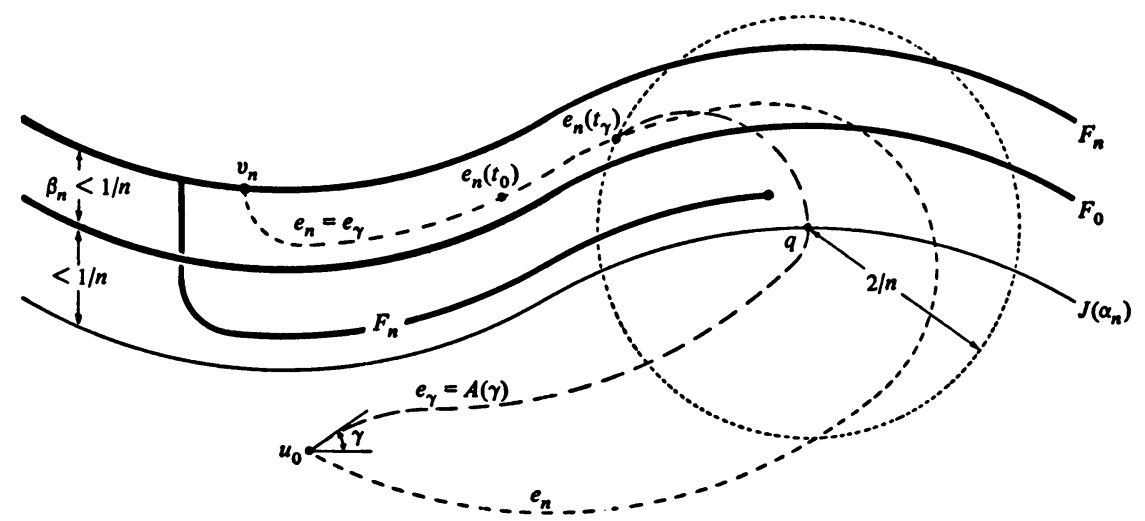

FIGURE 2

By (1), (2), and (3), $e_{\gamma}$ and $t_{\gamma}$ exist for some $\gamma$.

(6) Set $t_{0}=\sup \left\{t_{\gamma}: 0 \leqslant \gamma<2 \pi\right\}$, and choose a fixed angle $\gamma$ such that $e_{\gamma}$ exists and such that $e_{n}\left(\left[t_{\gamma}, t_{0}\right]\right)$ has diameter less than $1 / n$. For this fixed $\gamma$, denote the intersection of $A(\gamma)$ with $J\left(\alpha_{n}\right)$ by $q$.

(7) Choose points $q^{\prime}, q^{\prime \prime} \in J\left(\alpha_{n}\right)$ such that $\left|q-q^{\prime}\right|,\left|q-q^{\prime \prime}\right|$, and $\left|q^{\prime}-q^{\prime \prime}\right|$ all lie between $5 / n$ and $15 / n$ and such that the arc $q^{\prime} q q^{\prime \prime}$ of $J\left(\alpha_{n}\right)$ has diameter less than $50 / n$. (Here and later in the proof, we might have to insist that $n$ be sufficiently large.)

By (1) and (3), we can find points $s^{\prime}, s^{\prime \prime} \in F_{n}$ and arcs $q^{\prime} s^{\prime}$ and $q^{\prime \prime} s^{\prime \prime}$ of diameter less than $2 / n$ whose interiors lie outside $J\left(\alpha_{n}\right)$ and inside $G_{n}$. By (5) and the construction of $e_{\gamma}$, the arcs $q^{\prime} s^{\prime}$ and $q^{\prime \prime} s^{\prime \prime}$ do not intersect $e_{\gamma}([0,1])$. Thus the arc $Q_{n}=s^{\prime} q^{\prime} q q^{\prime \prime} s^{\prime \prime}$ is a cross cut in $G_{n}$ of diameter less 
than $60 / n$. But the portion of $e_{\gamma}([0,1])$ from $q$ to $v_{n}$ has diameter greater than or equal to $\epsilon$. Thus for any $\delta>0$, we can choose $n$ large enough so that $\operatorname{diam}\left(Q_{n}\right)<\delta$. Hence

$$
\eta_{n}(\delta) \geqslant d\left(G_{n} \backslash Q_{n}\right) \geqslant \epsilon>0,
$$

and so clearly condition (2) of Definition 2.1 does not hold.

Proof THAT (b) IMPLIES (c). We shall sketch a proof modeled after that in [10, pp. 59-62]. We wish to show that $\left\{f_{n}: n \geqslant 1\right\}$ is equicontinuous on $C_{1}\left(=\operatorname{bdry}\left(B^{2}\right)\right.$ ). Suppose otherwise. Then by shifting to subsequences (but keeping the same notation), we can find distinct points $r_{n}$ and $s_{n}$ of $C_{1}$ $(n \geqslant 1)$ such that the sequences $\left\{r_{n}\right\}$ and $\left\{s_{n}\right\}$ both converge to some $r_{0} \in$ $C_{1}$, and such that $r_{n}$ and $s_{n}$ correspond to distinct prime ends $a_{n}$ and $b_{n}$ of $G_{n}$, where $\left\{a_{n}\right\}$ and $\left\{b_{n}\right\}$ converge (as sequences of prime ends-see Remark 2.3) to distinct prime ends $a_{0}$ and $b_{0}$ of $G_{0}$. One of the arcs with endpoints $r_{n}$ and $s_{n}$ is constricted to $r_{0}$ as $n$ tends to infinity, and denote the corresponding $H$-intervals in $F_{n}$ by $A_{n}$. Denote the complementary $H$-intervals in $F_{n}$ by $B_{n}(n \geqslant 1)$.

(1) We can label the $H$-intervals determined by $a_{0}$ and $b_{0}$ with $A_{0}$ and $B_{0}$ so that the sequences $\left\{A_{n}\right\}$ and $\left\{B_{n}\right\}$ converge in the sense of condition (b) to $A_{0}$ and $B_{0}$, respectively. (In this and other proofs, each numbered statement is an assertion whose proof is clear or follows the statement.)

For the labeling, choose a prime end $w_{0}$ in $F_{0}$ not equal to either $a_{0}$ or $b_{0}$. Condition (b) shows that $w_{0}$ is the limit of a sequence $\left\{w_{n}\right\}$ of prime ends. By taking a subsequence, we can assume that $w_{n} \in A_{n}$ or $w_{n} \in B_{n}$ for all $n$. We label the $H$-interval containing $w_{0}$ with $A_{0}$ in the first case and with $B_{0}$ in the second case.

In order to show that condition (b) holds for the sequence $\left\{A_{n}\right\}$ and for $A_{0}$, let $v_{0}$ be any prime end of $A_{0}$. By condition (b) in the original form, there is a sequence $\left\{v_{n} \in F_{n}\right\}$ converging to $v_{0}$. We claim that $v_{n} \in A_{n}$ for all but finitely many $n$.

In proving this last claim, we assume that $w_{0}$ (used in the labeling) lies in $A_{0}$, that $v_{0}$ is neither $a_{0}, w_{0}$, nor $b_{0}$, and that all prime ends come equipped with representing embeddings starting at $u_{0}$. It is not too hard to show that for sufficiently large $n$, we can assume that terminal portions of the images of representing embeddings for $a_{0}, b_{0}, v_{0}$, and $w_{0}$ are disjoint. For sufficiently large $n$, we construct a simply connected subdomain $G_{n}^{\prime}$ of $G_{n}$ using

(i) terminal portions of the embeddings for $a_{n}$ and $b_{n}$,

(ii) for $\alpha$ sufficiently close to 1 , that portion of the standard simple closed curve $J(\alpha)$ not intersecting the embeddings for $w_{n}$ or $v_{n}$ and terminating in 
intersections with the embeddings for $a_{n}$ and $b_{n}$, and

(iii) the $H$-interval $A_{n}$.

This construction uses the uniform convergence of the representing embeddings. Because $w_{n} \in A_{n}$, we have $u_{0} \in G_{n}^{\prime}$, since the embedding for $w_{n}$ starts at $u_{0}$ and does not meet bdry $\left(G_{n}^{\prime}\right)$. Using similar reasoning, we must have $v_{n} \in A_{n}$ for sufficiently large $n$, completing the proof of the claim.

To finish the proof of (1), we know that a sequence $\left\{v_{n} \in A_{n}\right\}$ must have a subsequence converging to some $v_{0} \in F_{0}$, and as above it is not too hard to show that $v_{0} \in A_{0}$. Similarly, we see that $\left\{B_{n}\right\}$ converges to $B_{0}$ in the sense of condition (b).

Choose a prime end $c_{0}$ in the interior of $A_{0}$ such that, as a point, $c_{0}$ is not an endpoint of $F_{0}$, i.e., $c_{0}$ does not have arbitrarily small neighborhoods whose boundaries intersect $F_{0}$ in a single point $[26$, p. 64]. (The non endpoints are dense in $F_{0}$.)

For each $\epsilon>0$, let $V(\epsilon)$ denote the open disk centered at $c_{0}$ with radius $\epsilon$, and let $W_{0}(\epsilon)$ denote the component of $V(\epsilon) \backslash F_{0}$ whose points can be connected to $c_{0}$ with an arc in $V(\epsilon) \backslash F_{0}$ representing $c_{0}$ as a prime end.

(2) There is an $\epsilon_{1}>0$ such that for all $\epsilon$ satisfying $0<\epsilon \leqslant \epsilon_{1}$, any neighborhood of $c_{0}$ inside $V(\epsilon)$ has boundary intersecting $F_{0}$ in more than one point.

It is clear that (2) is true because $c_{0}$ is not an endpoint of $F_{0}$. Using this same fact, it is not too hard to show (3) below.

(3) For all $\epsilon$ satisfying $0<\epsilon \leqslant \epsilon_{1}$, there are points arbitrarily close to $c_{0}$ outside $\operatorname{cl}\left(W_{0}(\epsilon)\right)$.

(4) There is an $\epsilon_{2} \leqslant \epsilon_{1}$ such that for all $\epsilon$ satisfying $0<\epsilon \leqslant \epsilon_{2}$ there is a positive integer $N(\epsilon)$ such that if $z_{0} \in W_{0}(\epsilon) \cap V(\epsilon / 2)$ and $n \geqslant N(\epsilon)$, then any prime end of $F_{n}$ representable with an arc from $z_{0}$ inside $V(\epsilon)$ must lie in $A_{n}$. (See Figure 3.)

If (4) were not true, then, starting with a fixed representing embedding $e_{0}$ for $c_{0}$, we can construct (taking another subsequence) a sequence $\left\{v_{n} \in B_{n}\right\}$ of prime ends with representing embeddings $e_{n}$ which agree with $e_{0}$ to within $1 / n$ of $c_{0}$. This sequence clearly converges to $c_{0}$, giving a contradiction to (1).

Choose a fixed $z_{0} \in W_{0}\left(\epsilon_{2}\right) \cap V\left(\epsilon_{2} / 2\right)$ satisfying (4), and hence also (3). For each $n \geqslant N(\epsilon)$, let $W_{n}(\epsilon)$ denote the component of $V(\epsilon) \backslash F_{n}$ containing $z_{0}$.

(5) There is an $\epsilon$ satisfying $0<\epsilon<\epsilon_{2}$, a $\delta$ satisfying $0<\delta \leqslant \epsilon / 2$, and a positive integer $N$ such that $z_{0} \in W_{0}(\epsilon) \cap V(\epsilon / 2)$, and such that there is a fixed arc $R$ of the circle $C=\left\{u:\left|u-z_{0}\right|=\delta\right\}$ which is disjoint from $\operatorname{cl}\left(W_{n}(\epsilon)\right)$ for all $n \geqslant N$. We assume that $R$ makes an angle of $2 \pi / k$ for some integer $k$. 


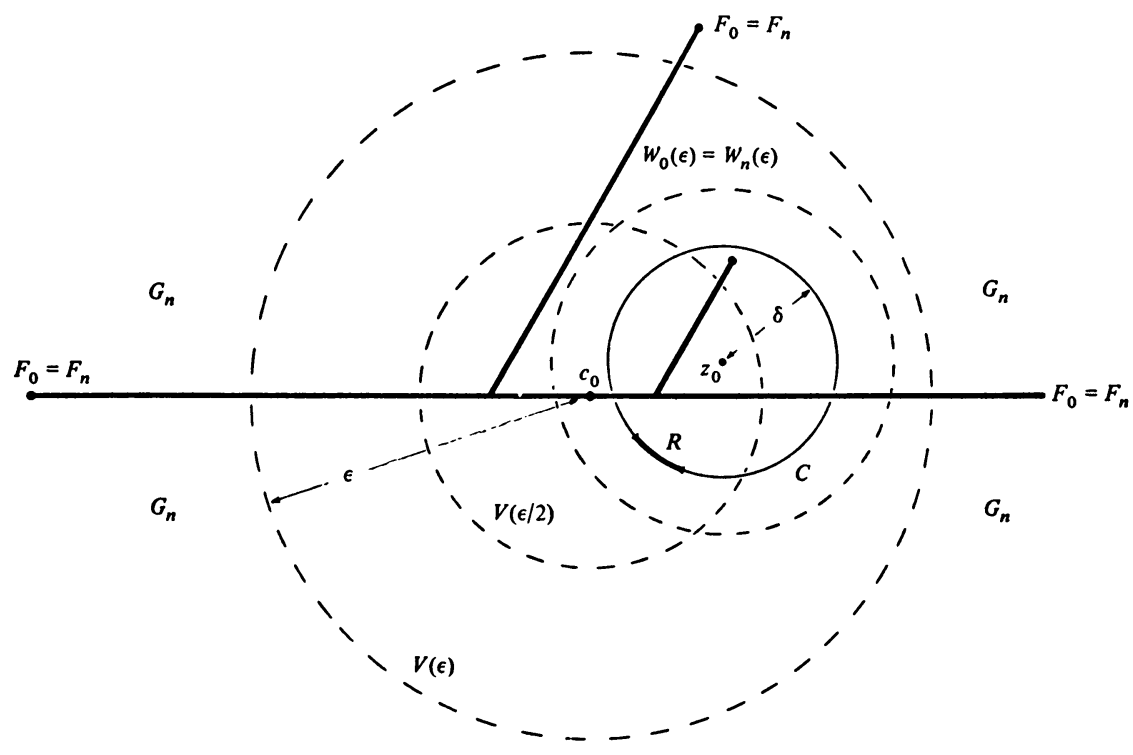

Figure 3

Choose $\epsilon<\epsilon_{2}$ such that $z_{0} \in W_{0}(\epsilon) \cap V(\epsilon / 2)$. (It is easy to choose such an $\epsilon$ after first choosing an arc from $z_{0}$ inside $W_{0}\left(\epsilon_{2}\right)$ which represents $c_{0}$ as a prime end.) Using (3), choose $\delta \leqslant \epsilon / 2$ and $R$ such that $R$ is disjoint from $\mathrm{cl}\left(W_{0}\left(\epsilon_{2}\right)\right)$. The problem we encounter with (5) is that $W_{n}(\epsilon)$ may be drastically larger than $W_{0}(\epsilon)$ for all $n$. Let $G(\epsilon)$ be the set of all points with a neighborhood contained in $W_{n}(\epsilon)$ for sufficiently large $n$. It is clear that $\operatorname{bdry}\left(W_{0}(\epsilon)\right)$ is disjoint from $G(\epsilon)$, so the kernel of $\left\{W_{n}(\epsilon)\right\}$ with respect to $z_{0}$ (which is the component of $G(\epsilon)$ containing $z_{0}$-see Definition 1.3) will be contained in $W_{0}(\epsilon)$. Now $G(\epsilon)$ may have other components outside $W_{0}(\epsilon)$, but since $\epsilon<\epsilon_{2}$, it is not too hard to see that $G(\epsilon)$ will be contained in $W_{0}\left(\epsilon_{2}\right)$. (By condition (b) and the Carathéodory convergence, a separate component of $G(\epsilon)$ can only occur if portions of $F_{n}$ extend arbitrarily closely to $\operatorname{bdry}(V(\epsilon))$, for increasing $n$.) It is now not hard to choose an $N$ so that (5) is true.

Set $g_{n}(u)=f_{n}^{-1}(u)-r_{0}$, for $u \in W_{0}(\epsilon)$ and $n \geqslant N$. Let $m_{n}$ be the supremum over all $w$ in $\operatorname{bdry}\left(W_{n}(\epsilon)\right)$ of the numbers $\lim \sup \left\{\left|g_{n}(u)\right|\right.$ : $\left.u \rightarrow w, u \in W_{n}(\epsilon)\right\}$. By (4), $m_{n}$ tends to 0 as $n$ tends to infinity. Using (5) and Lemma 1.7, we see that $\left|g_{n}\left(z_{0}\right)\right| \leqslant\left(m_{n} 2^{k-1}\right)^{1 / k}$. Thus as $n$ tends to infinitely ( $k$ fixed), $g_{n}\left(z_{0}\right)$ tends to zero. On the other hand, $g_{n}\left(z_{0}\right)$ must converge to $f_{0}^{-1}\left(z_{0}\right)-r_{0}$, i.e., $f_{0}^{-1}\left(z_{0}\right)=r_{0}$. This is true for infinitely many $z_{0} \in G_{0}$, so we have a contradiction. Thus the set of functions $\left\{f_{n}: n \geqslant 1\right\}$ is equicontinuous on $C_{1}$. 
The remainder of the proof follows as in [10]. In brief, a subsequence of $\left\{f_{n}\right\}$ converges uniformly on $C_{1}$. Hence $\left\{f_{n}\right\}$ converges pointwise on $C_{1}$, since otherwise there would be subsequences of $\left\{f_{n}\right\}$ converging to distinct analytic functions on $\operatorname{int}\left(B^{2}\right)$. It follows that $\left\{f_{n}\right\}$ converges uniformly on $C_{1}$ and hence on $B^{2}$, and clearly it converges to $f_{0}$.

ProOF THAT (c) IMPLIES (e). Without loss of generality, we can suppose that each $G_{n}$ contains the closed unit disk $B^{2}$ and that each $f_{n}$ fixes the origin. For each $n$, define $g_{n}: A^{2} \rightarrow A\left(f_{n}^{-1}\left(C_{1}\right), C_{1}\right)$ as in the proof that (c) implies (e) in Theorem 1.1, and suppose that each $g_{n}$ maps $(1,0)$ to $f_{n}^{-1}(1,0)$. Note that $h_{n}=f_{n} \circ g_{n}$ fixes the point $(1,0)$ and that $\left\{f_{n}^{-1}(1,0)\right\}$ converges to $f_{0}^{-1}(1,0)$. By condition (c) it is clear that the sequence of simple closed curves $\left\{f_{n}^{-1}\left(C_{1}\right)\right\}$ Fréchet converges to $f_{0}^{-1}\left(C_{1}\right)$. Results about mappings of annular regions between simple closed curves show that $\left\{g_{n}\right\}$ converges to $g_{0}$ uniformly on $A^{2}$. (See [11], [14], [16] and [22].) Thus $\left\{h_{n}\right\}$ converges to $h_{0}$ uniformly on $A^{2}$. For each $n$, define a retraction $\psi_{n}$ of $E^{2} \backslash \operatorname{int}\left(B^{2}\right)$ onto $E^{2} \backslash G_{n}$ as in the proof of Theorem 1.1. Arguing by contradiction, it is not hard to prove that the sequence $\left\{\psi_{n}\right\}$ converges to $\psi_{0}$ uniformly on $E^{2} \backslash \operatorname{int}\left(B^{2}\right)$.

Proof that (e) impleses (d). This is trivial.

Proof that (d) imples (a). Assume (d) is true and (a) is not true. From (d) it is easy to see that $\left\{G_{n}\right\}$ Carathéodory converges to $G_{0}$. If (a) is not true, then part (2) of Definition 2.1 must fail. Thus, for each $n$ there is a cross cut $Q_{n}$ of $G_{n}$ such that as $n$ tends to infinity, $\operatorname{diam}\left(Q_{n}\right)$ tends to zero, but $d\left(G_{n} \backslash Q_{n}\right)$ and $\operatorname{dist}\left(Q_{n}, u_{0}\right)$ do not tend to zero. The retraction $\varphi_{n}$ fixes the endpoints of $Q_{n}$, but moves some interior point a distance which does not tend to zero as $n$ tends to infinity. This is clearly a contradiction to the uniform convergence of $\left\{\varphi_{n}: n \geqslant 1\right\}$. Q.E.D.

As in $\S 1$, we shall need extensions of Theorem 2.2 to an arbitrary (second countable) 2-manifold $M^{2}$. For each nonnegative integer $n$, let $G_{n}$ be a precompact simply connected domain in $M^{2}$ with locally connected boundary $F_{n}$ consisting of more than one point. For each $n$, let $f_{n}: B^{2} \rightarrow G_{n} \cup F_{n}$ be the mapping given by Theorem 1.5, normalized as in the discussion preceding Theorem 1.6. Using the universal covering space as in the proof of Theorem 1.5, we get the following result.

2.4. THEOREM. The five conditions of Theorem 2.1 make sense and are equivalent in this more general setting.

3. The space of deformation retractions. In this section and the next, we study the space $R\left(M^{2}\right)$ of retractions of an arbitrary compact 2-manifold $M^{2}$, where the compact-open (= sup-metric) topology is used. The continuity property 
of $\S 2$ was developed as a tool for the study of $R\left(M^{2}\right)$. For material on retractions, see [5] and [13].

There is a number $\epsilon>0$ such that any two selfmaps of $M^{2}$ within a distance $\epsilon$ are homotopic. (An argument can be given using geodesics.) Also, it will be clear from work below that the space $D\left(M^{2}\right)$ of deformation retractions of $M^{2}$ is pathwise connected. Hence $D\left(M^{2}\right)$ is a component in $R\left(M^{2}\right)$. (See [21] and [22].) We can use Theorem 1.1 to construct a canonical path in $D\left(M^{2}\right)$ from any deformation retraction to the identity map on $M^{2}$, and then Theorem 2.2 implies the following principal result of this section.

3.1. THEOREM. For any compact 2-manifold $M^{2}$, the space $D\left(M^{2}\right)$ of deformation retractions of $M^{2}$ is contractible in itself.

As a prototype for the construction used in this section and in the proofs of Theorem 4.2 and Lemma 5.3, we have the following, due in its first forms to Alexander [2] and Borsuk [4].

3.2. REMARK [21, p. 320]. Let $M$ be a manifold with boundary $\partial M$ and let $\partial M \times[0,2] \subset M$ be a collar of the boundary. For $t \in[0,1]$, let $\rho_{t}$ be the retraction of $M$ which is the identity outside $\partial M \times[0, t)$ and projects $\partial M \times[0, t]$ to $\partial M \times\{t\}$. Let $h_{t}: M \rightarrow \rho_{t}(M)$ be the homeomorphism given by the identity outside $\partial M \times[0,2)$ and by mapping $\partial M \times[0,2]$ linearly to $\partial M \times[t, 2]$. Then the homotopy $\Theta_{t}$ given by $\Theta_{t}(\varphi)=$ $h_{t} \circ \varphi \circ h_{t}^{-1} \circ \rho_{t}$ provides a deformation of $R(M)$ in itself such that the image of $\Theta_{1}$ consists of retractions of $M$ whose images do not meet $\partial M$.

If $M$ is compact, we can vary the amount a retraction is moved according to the distance it overlaps onto $\partial M \times[0,1)$. In this way, we can prove the following result.

3.3. TheOREM (ElBowroom CONSTRUCTION). If $M$ is a compact manifold with boundary $\partial M$ and if $\partial M \times[0,1] \subset M$ is a collar, then there is a strong deformation retraction of $R(M)$ onto the space of retractions of $M$ with images in $M \backslash(\partial M \times[0,1))$.

Proof of.THEOREM 3.1. The proof is similar to the proof that (c) implies (e) in Theorems.1.1 and 2.2. Let $L^{2}$ be the manifold obtained from $M^{2}$ by filling in each hole with an open disk. We shall show in Theorem 3.4 below that for any $\varphi$ in $D\left(M^{2}\right), L^{2} \backslash \operatorname{im}(\varphi)$ consists of a simply connected domain containing each of the open disks. We work in the universal covering space of $L^{2}$ (which we assume to be $E^{2}$; if it is $S^{2}$, the proof is similar), and project the construction down into $L^{2}$, handling each hole separately. Thus without loss of generality, we assume that $M^{2}$ is $E^{2} \backslash \operatorname{int}\left(B^{2}\right)$ and that $L^{2}$ is $E^{2}$. (This is not compact, but all functions will be fixed outside a bounded portion of $E^{2}$.) 
Given a deformation retraction $\varphi$ of $E^{2} \operatorname{lint}\left(B^{2}\right)$, we can use Remark 3.2 or Theorem 3.3 to assume that $\operatorname{im}(\varphi)$ is disjoint from the boundary (which is $C_{1}$ here), and we can assume that $\operatorname{im}(\varphi)$ is bounded in $E^{2}$.

First, we show how to produce a canonical deformation retraction $\xi_{1}$ of $E^{2} \operatorname{lint}\left(B^{2}\right)$ onto $E^{2} \lim (\varphi)$, such that each stage of the deformation is itself a deformation retraction of $E^{2} \operatorname{lint}\left(B^{2}\right)$. As in the proof of Theorem 1.1, let $h$ be the unique continuous function from $A^{2}=A\left(C_{1}, C_{2}\right)$ onto the closure of $E^{2} \backslash\left(\operatorname{im}(\varphi) \cup B^{2}\right)$ which is a radical contradiction of a map conformal on the interior and which fixes the point $(1,0) \in C_{1}$. For each $t \in[0,1]$, let $\lambda_{t}$ be the retraction of $A^{2}$ onto $A\left(C_{1+t}, C_{2}\right)$ which projects $A\left(C_{1}, C_{1+t}\right)$ radially onto $C_{1+t}$. Define a retraction $\xi_{t}$ of $E^{2} \backslash \operatorname{int}\left(B^{2}\right)$ by setting $\xi_{t}=h \circ \lambda_{t} \circ h^{-1}$ on the closure of $E^{2} \backslash\left(\operatorname{im}(\varphi) \cup B^{2}\right)$ and letting $\xi_{t}$ fix the points of $\operatorname{im}(\varphi)$. For each $t \in$ $[0,1], \xi_{t}$ is clearly a retraction (it is idempotent) and clearly continuous (the case $t=1$ is contained in the proof of Theorem 1.1). To prove continuity of $\xi_{t}$ in $t$ when $t=1$, show that, given $\epsilon>0$, there is a number $r<1$ such that $\sup \left\{\left|\xi_{t}(u)-\xi_{1}(u)\right|: u \in E^{2} \backslash \operatorname{int}\left(B^{2}\right)\right\}<\epsilon$ for all $t$ satisfying $r<t \leqslant 1$. This is not hard to show using the facts that $h$ is uniformly continuous on $A^{2}$ and that $\lambda_{t}$ moves the points of this annulus radially out towards $C_{2}$.

Thus, $\xi_{t}$ gives a canonical path in the space of deformation retractions of $E^{2} \operatorname{lint}\left(B^{2}\right)$ from the identity to a canonical retraction $\xi_{1}$ of $E^{2} \operatorname{lint}\left(B^{2}\right)$ onto $\operatorname{im}(\varphi)$. Setting $\sigma_{t}=\varphi \circ \xi_{t}$ (a retraction for each $t$ because $\operatorname{im}\left(\xi_{t}\right)$ contains $\operatorname{im}(\varphi)$ ), we obtain a path from $\varphi$ to $\xi_{1}=\sigma_{1}$, and $\xi_{1-t}$ gives a path from $\xi_{1}$ to the identity on $E^{2} \operatorname{lint}\left(B^{2}\right)$. Theorem 2.2 shows that these canonical paths give the desired contraction of $D\left(E^{2} \operatorname{int}\left(B^{2}\right)\right)$. Q.E.D.

The next result characterizes the deformation retracts of $M^{2}$ and thus generalizes a theorem of Borsuk [3], [5, p. 132].

3.4. ThEOREM. Let $M^{2}$ be any compact 2-manifold and let $m$ be the number of its boundary curves. Let $L^{2}$ be a compact 2-manifold without boundary containing disjoint open disks $D_{j}(1 \leqslant j \leqslant m)$ such that $M^{2}=L^{2} \backslash \bigcup\left\{D_{j}\right\}$. Let $R$ be a subset of $M^{2}$ such that

(1) $R$ is connected, locally connected and closed, and

(2) $G=L^{2} \backslash R$ consists of simply connected components $G_{j}$ with $D_{j} \subset G_{j}$ $(1 \leqslant j \leqslant m)$.

Then we can conclude that

(i) $R$ is the image of a deformation retraction $\xi$ of $M^{2}$, and

(ii) if $R$ is the image of a retraction $\varphi$ of $M^{2}$, then $\varphi$ is a deformation retraction, and there is a canonical deformation in $D\left(M^{2}\right)$ from $\varphi$ to the identity.

Conversely, let $M^{2}$ and $L^{2}$ be as above, and let $\varphi$ be a deformation 
retraction of $M^{2}$. Then $R=\mathrm{im}(\varphi)$ satisfies (1) and (2) above.

Proof. If $R \subset M^{2}$ satisfies (1) and (2) above, then (i), (ii) follow from the proof of Theorem 3.1.

For the converse, let $\varphi \in D\left(M^{2}\right)$. Condition (1) of the theorem is clear. Suppose the universal covering space of $L^{2}$ is $E^{2}$, with covering projection $p$, and let $N^{2}$ denote the plane less the infinite collection of disjoint open disks $p^{-1}\left(D_{j}\right)$, for $1 \leqslant j \leqslant m$. The space $N^{2}$ is a covering space of $M^{2}$, and since $\varphi$ is a deformation retraction, it will lift to a deformation retraction $\psi$ of $N^{2}$ [19].

First, suppose $G=L^{2} \backslash R$ contains a component $H$ which is not simply connected. Then we can produce a simple closed curve $J$ in $H$ which is not contractible in $H$, hence also not contractible in $L^{2}$, since $\operatorname{im}(\varphi)$ is connected. Thus $p^{-1}(J)$ will consist of an infinite collection of closed embeddings of $E^{1}$ in $E^{2}$, each of which separates $E^{2}$. This contradicts the fact that $\operatorname{im}(\psi)$ is connected. Hence each component of $G$ is simply connected.

Next, suppose $H$ is a component of $G$ which does not contain any of the disks $D_{j}$. Let $H^{\prime}$ be a component of $p^{-1}(H)$. Since $H$ is simply connected, $H^{\prime}$ will be open and bounded. The retraction $\psi$, when restricted to $\operatorname{cl}\left(H^{\prime}\right)$, would map $\operatorname{cl}\left(H^{\prime}\right)$ into $E^{2} \backslash H^{\prime}$ and fix the points on the boundary. Using the Brouwer fixed point theorem [5, p. 12], it is easy to see that there can be no such map.

Finally, if any component of $G$ contains more than one of the disks $D_{j}$, then we could use the first part of this theorem to construct a deformation retraction of $L^{2}$ with $k$ holes $(k<m)$ onto $R$. Thus $L^{2}$ with $k$ holes would have the same homotopy type as $L^{2}$ with $m$ holes, which is not true.

The case in which the universal covering space of $L^{2}$ is $S^{2}$ can be handled as above by embedding $L^{2}$ minus a hole in $E^{2}$ and arguing separately if $m=0$. Q.E.D.

4. The space of nullhomotopic retractions. The nullhomotopic retractions of a compact 2-manifold $M^{2}$ are exactly those with contractible image, and the space of these retractions, with the compact-open (= sup-metric) topology, is a component in the space $R\left(M^{2}\right)$ of all retractions of $M^{2}$. (As noted in [22, pp. 611-612], this follows because any two sufficiently close selfmaps of $M^{2}$ must be homotopic and because the space of nullhomotopic retractions is pathwise connected, as will be shown below.)

4.1. Definition. Let $\Lambda$ be the canonical embedding of $M^{2}$ into its space of retractions which maps each point of $M^{2}$ to the constant retraction to that point. Denote by $L\left(M^{2}\right)$ the component containing the image of $\Lambda$, and restrict the range of $\Lambda$ to $L\left(M^{2}\right)$. Finally, let $e v: L\left(M^{2}\right) \rightarrow M^{2}$ be the evaluation map 
which takes a retraction to the retraction evaluated at a basepoint of $M^{2}$.

It is clear that $L\left(M^{2}\right)$ is exactly the space of nullhomotopic retractions of $M^{2}$.

4.2. THEOREM. For any compact 2-manifold $M^{2}$, the embedding $\Lambda$ : $M^{2} \rightarrow L\left(M^{2}\right)$ is a homotopy equivalence with homotopy inverse the evaluation map ev. Thus, the space $L\left(M^{2}\right)$ of nullhomotopic retractions of $M^{2}$ has the same homotopy type as $M^{2}$.

In outline, the proof is similar to the proofs in [21] and [22], except that here instead of Michael's selection theorem, we use the following result, which will also be needed in $\S 6$.

4.3. LeMma (EXISTENCE OF CROSS SeCtions [20, p. 55]). Let $(X, \tau, Y)$ be a locally trivial fibre space $[20$, p. 3], $[19$, p. 90] such that $Y$ is separable metric and the fibre $F$ is an absolute retract $[13$, p. 95]. Then any partial cross section defined on a (possibly empty) closed subspace $A$ of $Y$ extends to a cross section, i.e., a map $e: Y \rightarrow X$ such that $\tau \circ e$ is the identity on $Y$.

Using Theorem 3.3, we can assume that each retraction in $L\left(M^{2}\right)$ has image missing $\partial M^{2}$, and this assumption will hold for the rest of this section. Let $S$ denote the collection of all simple closed curves $J$ in $M^{2}$ which bound a closed disk $B(J)$ in $M^{2}$ and which do not meet $\partial M^{2}$. (The disk $B(J)$ is uniquely specified unless $M^{2}$ is the 2-sphere.) The set $S$, with the Fréchet topology (Definition 2.1), becomes a separable metric space [22], and using the methods of [14], it is not hard to see that $S$ is an absolute neighborhood retract (ANR). (In [14, Lemma 11], it is shown that the space of embeddings of $B^{2}$ into $E^{2}$ which are analytic on $\operatorname{int}\left(B^{2}\right)$ is an ANR. This space has as a retract the subspace consisting of embeddings fixing the origin and having positive derivative at the origin. The space $S$ is locally homeomorphic to the latter space.)

For any $\varphi \in L$, let $S(\varphi)$ denote the space of all $J \in S$ such that $\operatorname{im}(\varphi)$ is contained in $\operatorname{int}(B(J))$, the interior of the disk which $J$ bounds. Each $S(\varphi)$ is nonempty [22, Lemma 1], is contractible in itself [22, Lemma 2(b)], and is clearly open in $S$. Hence $[13$, p. 96] $S(\varphi)$ is an absolute retract for each $\varphi$. Let $B$ be the subspace of $L \times S$ consisting of all $(\varphi, J)$ such that $J \in S(\varphi)$, and let $\tau: B \rightarrow L$ be the restriction of the projection onto the first coordinate.

4.4. Lemma. The triple $(B, \tau, L)$ is a locally trivial fibre space with fibre the absolute retract $S\left(\Lambda\left(u_{0}\right)\right)$, where $u_{0}$ is a basepoint of $M^{2}$.

Proof. Let $\varphi_{0} \in L$. Let $U$ be a neighborhood of $\varphi_{0}$ in $L$ small enough so that for fixed $J_{0} \in S$ and some $\epsilon_{0}>0$, we have, for all $\varphi \in U$, 
$\operatorname{im}(\varphi) \subset B\left(J_{0}\right)$ and $\operatorname{dist}\left(\operatorname{im}(\varphi), J_{0}\right)>\epsilon_{0}$. For each $\varphi \in U$, let $g_{\varphi}$ be a homeomorphism of $B\left(J_{0}\right) \backslash \operatorname{im}(\varphi)$ onto $B\left(J_{0}\right) \backslash i m\left(\varphi_{0}\right)$ which is a radial contraction of a map conformal on the interior. It is easy to adjust $g_{\varphi}$ to a unique homeomorphism (still termed $g_{\varphi}$ ) which is the identity on $J_{0}$. (See [21] and [16, p. 45].) Then extend $g_{\varphi}$ to $M^{2} \backslash \operatorname{im}(\varphi)$ by the identity map. The map $g_{\varphi}$ induces a homeomorphism of $S(\varphi)$ onto $S\left(\varphi_{0}\right)$, and this in turn induces a homeomorphism $h$ of $\tau^{-1}(U)$ onto $U \times S\left(\varphi_{0}\right)$, given by $h(\varphi, K)=\left(\varphi, g_{\varphi}(K)\right)$. The continuity of $h$ and $h^{-1}$ follows from Theorem 2.2, using the technique in the proof that (c) implies (e). Because $L$ is pathwise connected (as will follow from work below), it is clear that the fibres are all homeomorphic to $S\left(\Lambda\left(u_{0}\right)\right)$. Q.E.D.

Proof of Theorem 4.2. By Lemma 4.3 (with $A$ empty), there is a cross section $e: L \rightarrow B$, and if $\tau_{2}$ is the projection onto the second coordinate, then for each $\varphi \in L, \tau_{2} \circ e(\varphi)$ is a canonical simple closed curve bounding a disk which contains $\operatorname{im}(\varphi)$ in its interior. (We are still assuming that $\operatorname{im}(\varphi)$ misses $\partial M^{2}$, for each $\varphi \in L$.)

The rest of the proof is similar to that in $[22$, p. 612], but we give a sketch here. For each $\varphi \in L$, let $f_{\varphi}: B\left(\tau_{2} \circ e(\varphi)\right) \rightarrow B^{2}$ be a conformal map which takes $\varphi\left(u_{0}\right)$ to the origin. The map $f_{\varphi}$ is determined only up to a rotation and possibly a reflection (if $M^{2}$ is not orientable), but the construction which follows is independent of rotations and reflections. The map $f_{\varphi} \circ \varphi \circ f_{\varphi}^{-1}$ is a retraction of $B^{2}$, and thus [21, Theorem 1.1] is homotopic to the constant retraction to the origin. The homotopy is similar to that in Remark 3.2, and is given by

$$
\psi_{t}=h_{t} \circ f_{\varphi} \circ \varphi \circ f_{\varphi}^{-1} \circ h_{t}^{-1} \circ \rho_{t},
$$

where $\rho_{t}$ projects $A\left(C_{1-t}, C_{1}\right)$ radially to $C_{1-t}$, and $h_{t}$ is a radial homeomorphism of $B^{2}$ onto $\rho_{t}\left(B^{2}\right)$. We define a retraction $\varphi_{t}$ piecewise on $M^{2}$ for each $t$. Set

$$
\varphi_{t}(x)=f_{\varphi}^{-1} \circ \psi_{t} \circ f_{\varphi}(x), \text { for } x \in B\left(\tau_{2} \circ e(\varphi)\right)
$$

and

$$
\varphi_{t}(x)=f_{\varphi}^{-1} \circ h_{t} \circ f_{\varphi} \circ \varphi(x), \text { for } x \in M^{2} \backslash \operatorname{int}\left(B\left(\tau_{2} \circ e(\varphi)\right)\right) \text {. }
$$

These definitions agree on the intersections of the domains, which is the curve $\tau_{2} \circ e(\varphi)$, so together they define a homotopy from $\varphi$ to $\Lambda\left(f_{\varphi}^{-1}(0,0)\right)=$ $\Lambda\left(\varphi\left(u_{0}\right)\right)=\Lambda \circ e v(\varphi)$. Theorem 2.2 shows that this defines a homotopy from the identity map on $L$ to the map $\Lambda \circ e v$. Clearly $e v \circ \Lambda$ equals the identity map on $M^{2}$. Q.E.D. 
4.5. REMARK. As in [22], Theorem 4.2 generalizes to an arbitrary (second countable) 2-manifold $M^{2}$, provided $M^{2}$ is given a metric in which it is complete, and provided we use the sup-metric topology on the space of retractions of $M^{2}$.

5. The space of retracts. In this section we consider the set of retracts (= images of retractions) of a compact 2-manifold $M^{2}$.

5.1. Definition. Let $\mathbf{R}\left(M^{2}\right), \mathrm{D}\left(M^{2}\right)$, and $\mathrm{L}\left(M^{2}\right)$ denote, respectively, the set of retracts of $M^{2}$, deformation retracts of $M^{2}$, and retracts of $M^{2}$ which are images of elements in $L\left(M^{2}\right)$. (Thus $L\left(M^{2}\right)$ consists of the set of compact absolute retracts in $M^{2}$ [13].) We give $\mathbf{R}\left(M^{2}\right)$ the quotient topology determined by the natural projection im: $R\left(M^{2}\right) \rightarrow \mathbf{R}\left(M^{2}\right)$ which maps a retraction to its image. The sets $\mathrm{D}\left(M^{2}\right)$ and $\mathbf{L}\left(M^{2}\right)$ are given topologies as subspaces of $\mathbf{R}\left(M^{2}\right)$.

5.2. REMARK. This topology on $\mathbf{R}\left(M^{2}\right)$ is strictly larger (= more open sets) than that of the Hausdorff metric. This follows because Fréchet convergence implies convergence in the Hausdorff metric, while simple examples show that the reverse implication is not true. (Since our retracts are compact, convergence in the Hausdorff metric is the same as the concept of "convergence" in [26, p. 10], i.e., the limit superior and the limit inferior are equal. See [12] and [17, pp. 50-58].)

For the statement of the following useful lemma, note that we define a submanifold of $M^{2}$ to be a compact subset which is a 2-manifold.

5.3. Lemma. A subset $R \subset M^{2}$ is a retract of $M^{2}$ if and only if there is a submanifold $N^{2}$ of $M^{2}$ such that

(i) $R$ is a deformation retract of $N^{2}$, and

(ii) $N^{2}$ is a retract of $M^{2}$.

Proof. If (i) and (ii) hold, then clearly $R$ is a retract of $M^{2}$. Suppose that $R$ is a retract of $M^{2}$. For now, we shall assume that $R$ is disjoint from $\partial M^{2}$. Given $u_{0} \in \operatorname{bdry}(R)$ and given an embedding $e_{0}: I=[0,1] \rightarrow M^{2}$ representing $u_{0}$ as a prime end (of the first kind, since $R$ is locally connected), we can construct a continuous function $E: I \times I \rightarrow M^{2}$ with the following properties:

(1) $E(t, 0)=e_{0}(t)$, for all $t \in I$,

(2) for each $t \in I, E(\cdot, t)$ is an embedding representing a prime end of $R$, and

(3) $E$ restricted to $[0,1) \times I$ is an embedding into $M^{2} \backslash R$.

Two functions $E$ and $E^{\prime}$ satisfying (2) and (3) overlap properly if exactly one of $E(t, 1)=E^{\prime}(t, 0)$ or $E(t, 0)=E^{\prime}(t, 1)$ holds, for all $t \in I$, and 
otherwise the images under $E$ and $E^{\prime}$ of $[0,1) \times I$ are disjoint.

We now construct finitely many mappings $E_{1}, E_{2}, \cdots, E_{n}$ satisfying (2) and (3) above and satisfying

(4) $E_{i}([0,1) \times(0,1))$ is disjoint from $E_{j}([0,1) \times(0,1))$ unless $i=j$,

(5) each $E_{i}$ overlaps properly with exactly two others (in the two ways above), and

(6) each prime end of $M^{2} \backslash R$ is represented by $E_{i}(\cdot, t)$ for some $i$ and $t$.

The compactness and local connectedness of $\operatorname{bdry}(R)$ insure that this construction may be carried out. Because $R$ is an ANR, we see that $M^{2} \backslash R$ can only have finitely many components. (We are still assuming that $R$ is disjoint from $\partial M^{2}$.) This fact is proved for the case $M^{2}=E^{2}$ in [5, p. 138] . It follows that $R$ and the images of the $E_{i}$ must form a compact 2-manifold with boundary the union of the arcs $E_{i}(0,1)$. Using the technique in the proof of Theorem 3.1, it is easy to produce a deformation retraction from this manifold onto $R$.

In order to prove condition (ii) of the lemma, we define the 2-manifold $N^{2}$ to be $N^{2}=R \cup\left(\bigcup_{i=1}^{n} E_{i}([2 / 3,1) \times I)\right)$. Let $\varphi$ be a retraction of $M^{2}$ with image $R$. From $\varphi$, we shall construct a retraction $\psi$ of $M^{2}$ with image $N^{2}$.

In the above work, we have constructed a half-open collar $[0,1) \times \partial N^{2}$ given by a continuous function $p: I \times \partial N^{2} \rightarrow M^{2}$ which is a homeomorphism except on $\{1\} \times \partial N^{2}$, where it provides a one-to-one correspondence between points and prime ends of $M^{2} \backslash R$. In this notation, $N^{2}$ is $R \cup\left([2 / 3,1) \times \partial N^{2}\right)$ and $\partial N^{2}$ is the same as $\{2 / 3\} \times \partial N^{2}$.

We define the retraction $\psi$ piecewise on the following four closed subsets of $M^{2}:$ (i) $N^{2}$, (ii) $[1 / 3,2 / 3] \times \partial N^{2}$, (iii) $[0,1 / 3] \times \partial N^{2}$, and (iv) $M^{2} \backslash\left(N^{2} \cup(0,2 / 3) \times \partial N^{2}\right)$. On $N^{2}$, we take $\psi$ equal to the identity map, and on the subset in (iv), we set $\psi=\varphi$. For the subset in (ii), we map [1/3, $2 / 3$ ] linearly onto $[2 / 3,1]$ (sending $1 / 3$ to 1 and fixing $2 / 3$ ) and project using $p$, so that $[1 / 3,2 / 3] \times \partial N^{2}$ is projected by $\psi$ onto $([2 / 3,1) \times$ $\left.\partial N^{2}\right) \cup \operatorname{bdry}(R)$, which is a subset of $N^{2}$. For the subset in (iii), map $[0,1 / 3]$ linearly onto $[0,1]$ (fixing 0 and sending $1 / 3$ to 1 ), project by $p$ into $M^{2}$ and follow by $\varphi$ suitably restricted. (Thus $\psi$ takes $\{1 / 3\} \times \partial N^{2}$ onto $\operatorname{bdry}(R)$ and $\{0\} \times \partial N^{2}$ into $R$ by the map $\varphi$.) It is clear that these definitions agree on the intersections of the domains, so together they give a continuous map $\psi$, which is also clearly a retraction with image $N^{2}$. (See Figure 4.)

If $R$ meets $\partial M^{2}$, attach a collar tc $\partial M^{2}$ and carry out the above construction in such a way that if a point $(t, u) \in I \times \partial N^{2}$ belongs to $M^{2}$, then the whole interval $[t, 1) \times\{u\}$ belongs to $M^{2}$. We omit the details. Q.E.D. 
The following theorem shows that the quotient topology of Definition 5.1 is natural in other ways. Notice the similarities with Theorems 1.1 and 2.2. In the statement below, for each submanifold $N^{2}$ of $M^{2}$, let $L^{2}$ be the manifold obtained by filling in each hole of $N^{2}$ with an open disk.
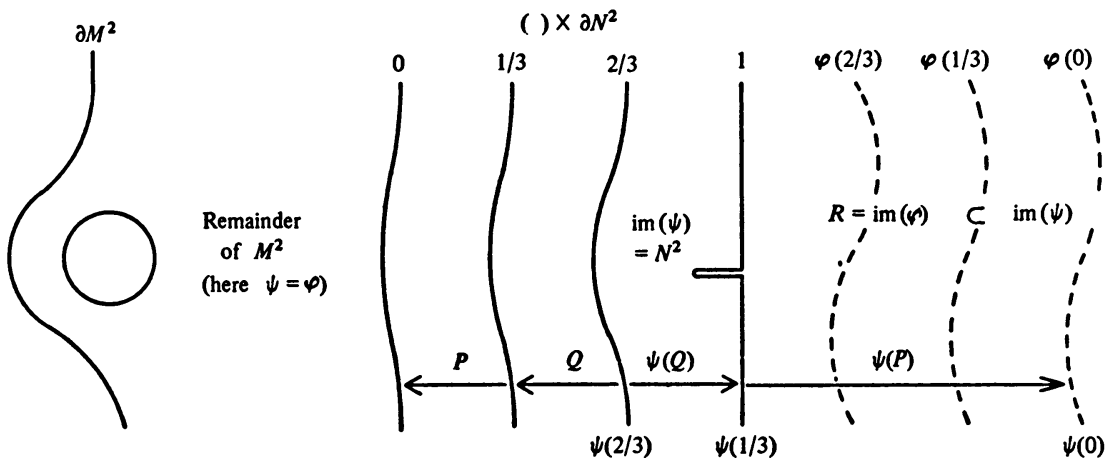

FIGURE 4

5.4. THEOREM (CONTINUITY PROPERTY FOR RETRACTS). Let $M^{2}$ be a compact 2-manifold, and for each nonnegative integer $n$, let $R_{n}$ be a retract of $M^{2}$. Then the following are equivalent.

(a) The sequence $\left\{R_{n}: n \geqslant 1\right\}$ converges to $R_{0}$ in the topology of Definition 5.1.

(b) There is a submanifold $N^{2}$ of $M^{2}$ which is a retract of $M^{2}$ and an integer $n_{0}$ such that for $n=0, n \geqslant n_{0}, N^{2}$ contains $R_{n}$ and $L^{2} \backslash R_{n}$ consists of simply connected domains, a domain $G_{n}$ for each hole of $N^{2}$, and for each hole, $\left\{\mathrm{bdry}\left(G_{n}\right): n \geqslant n_{0}\right\}$ Fréchet converges to $\operatorname{bdry}\left(G_{0}\right)$.

(c) With $N^{2}, n_{0}, L^{2}$ and $G_{n}$ as in (b), if $f_{n}: B^{2} \rightarrow \operatorname{cl}\left(G_{n}\right)$ is the corresponding map conformal on $\operatorname{int}\left(B^{2}\right)$ (normalized as in the discussion before Remark 1.6), then for each hole of $N^{2},\left\{f_{n}: n \geqslant n_{0}\right\}$ converges to $f_{0}$ uniformly on $B^{2}$.

(d) For each $n$, there is a retraction $\varphi_{n}$ of $M^{2}$ with image $R_{n}$ such that $\left\{\varphi_{n}: n \geqslant 1\right\}$ converges to $\varphi_{0}$ uniformly on $M^{2}$.

(e) There is a submanifold $N^{2}$ of $M^{2}$ which is a retract of $M^{2}$ and an integer $n_{0}$ such that for $n=0, n \geqslant n_{0}$, there are deformation retractions $\psi_{n}$ of $N^{2}$ with image $R_{n}$ such that $\left\{\psi_{n}: n \geqslant n_{0}\right\}$ converges to $\psi_{0}$ uniformly on $N^{2}$.

Proof. It is clear from the definition of quotient topology that (a) is equivalent to (d). The fact that (b) implies (c) is clear from Theorem 2.2. The proof that (c) implies (e) can be carried out using the construction in the proof of Theorem 3.1. Assuming (e), Theorem 3.4 shows that $L^{2} \backslash R_{n}$ consists of a simply connected domain $G_{n}$ for each hole of $N^{2}$. Since each $\psi_{n}(n=0$, $n \geqslant n_{0}$ ) is a deformation retraction of $N^{2}$, these maps lift to the covering space 
of $N^{2}$ given by taking the universal covering space of $L^{2}$ and omitting the lifted holes. This will be the plane with holes, and now Theorem 2.2 implies the Fréchet convergence of $\left\{\operatorname{bdry}\left(G_{n}\right): n \geqslant n_{0}\right\}$ to bdry $\left(G_{0}\right)$ for each hole of $N^{2}$. Hence we get (b). It remains to show that (d) and (e) are equivalent.

Assuming (e) again, let $\varphi: M^{2} \rightarrow N^{2}$ be a retraction and set $\varphi_{n}=$ $\psi_{n} \circ \varphi$. It is clear that (d) holds.

Finally, assuming (d), construct $N^{2}$ from $R_{0}$ as in the proof of Theorem 5.3. Choose $\epsilon$ less than $\operatorname{dist}\left(R_{0}, M^{2} \backslash N^{2}\right)$ such that any two selfmaps of $N^{2}$ within a distance $\epsilon$ are homotopic in $N^{2}$. Choose $n_{0}$ such that $n \geqslant n_{0}$ implies $\varphi_{n}$ is within $\epsilon$ of $\varphi_{0}$. Thus for all $n \geqslant n_{0}, R_{n} \subset N^{2}$ and $\varphi_{n N^{2}}$ is homotopic to $\left.\varphi_{0}\right|_{N^{2}}$ in $N^{2}$. Since $R_{0}$ is a deformation retract of $N^{2}$ and $\left.\varphi_{0}\right|_{N}$ is a retraction, we see by Theorem 3.4 that $\left.\varphi_{0}\right|_{N^{2}}$ is a deformation retraction. Hence $\left.\varphi_{n}\right|_{N^{2}}$ is a deformation retraction of $N^{2}$ for $n \geqslant n_{0}$. Setting $\psi_{n}=\left.\varphi_{n}\right|_{N} ^{2}$, we get (e). Q.E.D.

5.5. THEOREM. For any compact 2-manifold $M^{2}$, (i) the space $\mathbf{R}\left(M^{2}\right)$ of retracts of $M^{2}$ is an ANR (for metrizable spaces [13, Chapter 3]),

(ii) The space $\mathrm{D}\left(M^{2}\right)$ of deformation retracts of $M^{2}$ is an $A R$, and

(iii) The space $\mathbf{L}\left(M^{2}\right)$ of compact $A R$ subsets of $M^{2}$ is an $A N R$ with a homeomorphic copy of $M^{2}, \operatorname{im}\left(\Lambda\left(M^{2}\right)\right)$, as a deformation retract, and thus $\mathrm{L}\left(M^{2}\right)$ has the same homotopy type as $M^{2}$.

PRoof. Let $R$ be a retract of $M^{2}$ and choose $N^{2}$ as in Theorem 5.4. Suppose $L^{2}=N^{2} \cup\left(\bigcup\left\{D_{j}: 1 \leqslant j \leqslant m\right\}\right)$ and $L^{2} \backslash R=\bigcup\left\{G_{j}: 1 \leqslant j \leqslant m\right\}$ are as in Theorem 3.4. If $R$ is disjoint from bdry $\left(D_{j}\right)$ for all $j$ and if $\operatorname{bdry}\left(G_{j}\right)$ is disjoint from $\operatorname{bdry}\left(G_{k}\right)$ for $j \neq k$, then at the member $R$, the space $\mathbf{R}\left(M^{2}\right)$ is locally homeomorphic to a product of copies of the ANR $A\left(B^{2}, E^{2}\right)$ described below (one copy for each hole of $N^{2}$ ), and hence $[13$, p. 97] is locally an ANR at $R$. The rest of the proof is needed to take care of the fact that $R$ may not be nicely situated in $M^{2}$.

(1) Let $A^{\prime}\left(B^{2}, E^{2}\right)$ denote the space of maps of $B^{2}$ into $E^{2}$ which are conformal homeomorphisms on $\operatorname{int}\left(B^{2}\right)$. This space was shown to be an ANR in [14, Lemma 9].

(2) Let $A\left(B^{2}, E^{2}\right)$ denote the subspace of $A^{\prime}\left(B^{2}, E^{2}\right)$ consisting of maps which fix the origin and have positive derivative at the origin. This space is clearly a retract of $A^{\prime}\left(B^{2}, E^{2}\right)$, and hence $[13$, p. 97] is an ANR.

For $L^{2}$ as above (without boundary) and any $u_{1} \in L^{2}$, let $A\left(B^{2}, L^{2}\right)$. denote the space of maps of $B^{2}$ into $L^{2}$ which send the origin to $u_{1}$, are conformal homeomorphisms on $\operatorname{int}\left(B^{2}\right)$, and send the positive $x$-axis to a fixed direction at $u_{1}$. 
(3) The space $A\left(B^{2}, L^{2}\right)$ is an $r$-image of $A\left(B^{2}, E^{2}\right)$ and hence [5, $\mathrm{p}$. 87] is an ANR. Also it follows from Theorem 2.2 that $A\left(B^{2}, L^{2}\right)$ is homeomorphic to the space $\mathbf{D}_{0}\left(L^{2} \backslash\left\{u_{1}\right\}\right)$ of those deformation retracts of $L^{2} \backslash\left\{u_{1}\right\}$ whose images miss some neighborhood of $u_{1}$.

To prove (3), we first describe a canonical shrinking of a map $f \in$ $A\left(B^{2}, E^{2}\right)$ by a factor $\beta(0<\beta \leqslant 1)$. Let $g_{\beta}$ be the obvious radial homeomorphism of $B^{2}$ onto $\beta \cdot B^{2}$ and set $\beta \cdot f: B^{2} \rightarrow L^{2}$ equal to $\left(\left.f\right|_{\beta \cdot B^{2}}\right) \circ g_{\beta}$. (Notice that for $\beta<1$, the image of $\beta \cdot f$ is a proper subset of the image of $\operatorname{int}\left(B^{2}\right)$ under $f$. Notice also that $\beta \cdot f \in A\left(B^{2}, E^{2}\right)$.) Let $p: E^{2} \rightarrow L^{2}$ be a conformal universal covering projection such that $p$ maps the origin to $u_{1}$ and the positive $x$-axis to the fixed direction at $u_{1}$. (The proof is similar if the universal covering space of $L^{2}$ is $S^{2}$ or $\operatorname{int}\left(B^{2}\right)$.) We now define an $r$-map from $A\left(B^{2}, E^{2}\right)$ onto $A\left(B^{2}, L^{2}\right)$ by sending $f$ to $p \circ(\beta \cdot f)$, where we take the largest value of $\beta$ for which the image map belongs to $A\left(B^{2}, L^{2}\right)$. (In order to construct a right inverse to our $r$-map, we need the facts that a simply connected domain in $L^{2}$ will lift under $p$ to homeomorphic copies, and that the functions in $A\left(B^{2}, E^{2}\right)$ and $A\left(B^{2}, L^{2}\right)$ are uniquely determined by the image of $\operatorname{int}\left(B^{2}\right)$.)

For distinct points $u_{1}, u_{2}, \cdots, u_{m} \in L^{2}$, define $A^{(m)}$ to be the space of all $m$-tuples $\left(f_{1}, f_{2}, \cdots, f_{m}\right)$ such that each $f_{j}$ belongs to $\mathrm{A}\left(B^{2}, L^{2}\right)$ (sending the origin to $u_{j}$ ) and such that $f_{j}\left(\operatorname{int}\left(B^{2}\right)\right)$ is disjoint from $f_{k}\left(\operatorname{int}\left(B^{2}\right)\right.$ ) for $j \neq k$.

(4) $A^{(m)}$ is a retract of the product of $m$ copies of the ANR $A\left(B^{2}, L^{2}\right)$, and hence $[13$, p. 97] is an ANR. (This can be proved using a shrinking procedure similar to that described after (3) for maps in $A\left(B^{2}, L^{2}\right)$.)

(5) The space $\mathrm{D}\left(N^{2}\right)$ is a retract of the space

$$
\mathbf{D}_{0}\left(L^{2} \backslash\left\{u_{1}, u_{2}, \cdots, u_{m}\right\}\right)
$$

consisting of deformation retracts missing a neighborhood of each $u_{j}$. (Here $u_{j} \in D_{j}$ for each $j$.) The latter space is homeomorphic to $A^{(m)}$, and hence $\mathrm{D}\left(N^{2}\right)$ is an ANR.

The retraction in (5) is not hard to construct using the method in the proof of Theorem 3.3. Here we use Remark 3.2 to move a retract a varying distance depending on how far it overlaps onto the disks $D_{j}$.

Finally, Theorem 5.4 shows that the space $\mathbf{R}\left(M^{2}\right)$ is locally homeomorphic to $\mathbf{D}\left(N^{2}\right)$ (for varying $N^{2}$ ), and hence [13, p. 98] $\mathbf{R}\left(M^{2}\right)$ is an ANR. Parts (ii) and (iii) of the theorem follow from corresponding statements about $D\left(M^{2}\right)$ and $L\left(M^{2}\right)$ (Theorems 3.1 and 4.2). Q.E.D.

6. Global forms of the continuity property. In Theorem 3.1, Lemma 4.4, and the proof of Theorem 5.5, we encountered global forms of Theorem 2.2. 
There are other similar results, and in this section we present two which seem interesting. Throughout, we assume that $M^{2}$ is an orientable Riemann surface [1] with a continuous nonvanishing vector field. Thus, $M^{2}$ must be noncompact, or with nonempty boundary, or must be the torus. (See [6] for other applications of vector fields on 2-manifolds.)

Let $F$ be the collection of all locally connected continua in $M^{2}$ such that each $F \in F$ is the boundary of a simply connected domain $G_{F}$, and give $F$ the topology of Fréchet convergence (Definition 2.1). The space $F$ is locally homeomorphic to a $G_{\delta}$ subset of a separable Banach space [14, pp. 278-279] and hence is locally complete. As in [22, Lemma 2(a)], we see that $F$ is a topologically complete separable metric space. The results which follow also hold for subspaces of $F$; for example, the space of simple closed curves bounding a disk.

Let $G$ be the subspace of $F \times M^{2}$ consisting of all $(F, u)$ such that $u \in G_{F}$, and let $\tau: G \rightarrow F$ be the restriction of the projection onto the first coordinate. Each fibre $\tau^{-1}(F)$ is homeomorphic to $\operatorname{int}\left(B^{2}\right)$ and hence is an AR. It follows from Theorem 2.2 that $(G, \tau, F)$ is a locally trivial fibre space. Thus by Lemma 4.3, there is a cross section $e: F \rightarrow G$. If $\tau_{2}$ is the projection onto the second coordinate, then $\tau_{2} \circ e: F \rightarrow M^{2}$ allows us to select continuously a canonical point $u_{F}$ from $G_{F}$ for each $F \in F$.

6.1. Definition. For each $F \in F$, define $f_{F}: B^{2} \rightarrow G_{F} \cup F$ to be the canonical continuous surjection which is a conformal homeomorphism on $\operatorname{int}\left(B^{2}\right)$, which maps the origin to $u_{F}$, and which maps the direction of the positive $x$-axis to the direction given by the vector field.

Let $g$ be the homeomorphism of $F X \operatorname{int}\left(B^{2}\right)$ onto $G$ defined by $g(F, u)=\left(F, f_{F}(u)\right)$. Let $A$ denote the space of all maps $f: B^{2} \rightarrow M^{2}$ which are conformal homeomorphisms on $\operatorname{int}\left(B^{2}\right)$, and let $p: A \rightarrow F$ be the natural continuous projection which maps $f$ to the image of $C_{1}$ under $f$. We can now easily derive the next result, which might be called a "global form of the Schoenflies theorem."

6.2. THEOREM. Using notation above,

(i) The homeomorphism $g$ satisfies $\tau \circ g=\tau_{1}$, the projection onto the first coordinate, i.e., $(G, \tau, F)$ is equivalent to the trivial fibre space $\left(F \times \operatorname{int}\left(B^{2}\right), \tau_{1}, F\right)$ and

(ii) the injection from $F$ into $A$ which maps $F$ to $f_{F}$ is a continuous right inverse for the projection $p$.

One can see from Theorem 2.2 that the solutions to the Dirichlet problem on a Riemann surface for simply connected domains with locally connected boundaries change continuously if the boundary values change continuously and 
the boundaries change continuously in the topology of Frechet convergence (see [7]). For a global statement of the above, we need the vector field. Let $F$, $G_{F}$, and $f_{F}$ be defined as above and let $\bar{G}$ be the set of all $(F, u) \in F \times$ $M^{2}$ such that $u \in G_{F} \cup F$. For each $F \in F$, suppose there is given a continuous function $b_{F}: F \rightarrow E^{1}$ such that the function $B$ defined on $F$ by $B(F)=b_{F} \circ\left(\left.f_{F}\right|_{C_{1}}\right)$ is continuous, where the range of $B$ is the space of continuous real-valued functions on $C_{1}$ with the sup-metric topology. (For example, one could use any continuous real-valued function $b$ defined on the union of the members of $F$ and set $b_{F}=\left.b\right|_{F}$.) We now have a global form of a solution to the Dirichlet problem.

6.3. THEOREM. Using notation above, there is a unique continuous realvalued function $h$ defined on $\bar{G}$ such that for fixed $F \in F, h(F, \cdot)$ is harmonic on $G_{F}$ and agrees with $b_{F}$ on $F$.

Proof. For each $F \in F$, let $k_{F}: B^{2} \rightarrow E^{1}$ be continuous on $B^{2}$, harmonic on $\operatorname{int}\left(B^{2}\right)$ and agree with $b_{F} \circ\left(\left.f_{F}\right|_{C_{1}}\right)$ on $C_{1}$. Then $k_{F} \circ f_{F}^{-1}=$ $h_{F}$ is harmonic on $G_{F}$, agrees with $b_{F}$ on $F$, and is continuous on $G_{F} \cup$ $F$. We define $h(F, u)=h_{F}(u)$, for $F \in F$ and $u \in G_{F} \cup F$. Arguing by contradiction, we see that $h$ is continuous. Q.E.D.

ADDED IN PROOF. Robert Cauty of Paris, France has independently obtained the results of $\S 4$ using completely different methods.

\section{REFERENCES}

1. L. V. Ahlfors and L. Sario, Riemann surfaces, Princeton Math. Series, no. 26, Princeton Univ. Press, Princeton, N.J., 1960. · MR 22 \# 5729.

2. J. W. Alexander, On the deformation of an n-cell, Proc. Nat. Acad. Sci. U. S. A. 9 (1923), 406-407.

3. K. Borsuk, Einige Sätze über stetige Strekenbilder, Fund. Math. 18 (1932), 198-213.

4. - Concerning the set of retractions, Colloq. Math. 18 (1967), 197-201. MR 36 \#2126.

5. - Theory of retracts, Monografie Mat., Tom 44, PWN, Warsaw, 1967. MR 35 \#7306.

6. D. R. J. Chillingworth, Winding numbers on surfaces. I, Math. Ann. 196 (1972), 218-249.

7. R. Courant, Bemerkung zu meiner Note: “ ̈̈ber eine Eigenschaft der Abbildungsfunctionen bei konformer Abbildung," Gött. Nachr. (1922), 69-70.

8. D. Gaier, Über die konforme Abbildung veränderlicher Gebiete, Math. Z. 64 (1956), 385-424. MR 17, 1191.

9. C. Gattegno and A. Ostrowski, Représentation conforme à la frontière; domaines généraux, Mém. Sci. Math., no. 109, Gauthier-Villars, Paris, 1949. MR 11, 425.

10. G. M. Goluzin, Geometric theory of functions of a complex variable, GITTL, Moscow, 1952; English transl., Transl. Math. Monographs, vol. 26, Amer. Math. Soc., Providence, R. I., 1969. MR 15, 112; MR 40 \#308. 
11. M.-E. Hamstrom and E. Dyer, Regular mappings and the space of homeomorphisms on a 2-manifold, Duke Math. J. 25 (1958), 521-531. MR 20 \#2695.

12. F. Hausdorff, Mengenlehre, 3rd ed., de Gruyter, Berlin and Leipzig, 1935.

13. S.-T. Hu, Theory of retracts, Wayne State Univ. Press, Detroit, Mich., 1965. MR 31 \#6202.

14. R. Luke and W. K. Mason, The space of homeomorphisms on a compact twomanifold is an absolute neighborhood retract, Trans. Amer. Math. Soc. 164 (1972), $275-285$.

15. A. I. Markusevic, Theory of functions of a complex variable. Vol. III, GITTL, Moscow, 1950; English transl., Prentice-Hall, Englewood Cliffs, N. J., 1967 . MR 12, 87; MR 35 \#6799.

16. H. R. Morton, The space of homeomorphisms of a disc with $n$ holes, Illinois $\mathrm{J}$. Math. 11 (1967), 40-48. MR 34 \# 5066.

17. W. Rinow, Die innere Geometrie der metrischen Raume, Die Grundlehren der math. Wissenschaften, Band 105, Springer-Verlag, Berlin, 1961. MR 23 \#A1290.

18. W. Rudin, Real and complex analysis, McGraw-Hill, New York, 1966. MR 35 \#1420.

19. E. H. Spanier, Algebraic topology, McGraw-Hill, New York, 1966. MR 35 \# 1007.

20. N. E. Steenrod, The topology of fibre bundles, Princeton Math. Series, no. 14, Princeton Univ. Press, Princeton, N. J., 1951. MR 12, 522.

21. N. R. Wagner, The space of retractions of the 2-sphere and the annulus, Trans. Amer. Math. Soc. 158 (1971), 319-329. MR 43 \# 5484.

22. - The space of retractions of a 2-manifold, Proc. Amer. Math. Soc. 34 (1972), 609-614. MR 45 \#4350.

23. - A continuity property and surface topology, Bull. Amer. Math. Soc. 79 (1973), 1308-1311.

24. - Components of the space of retractions of the torus, Preliminary report, Notices Amer. Math. Soc. 20 (1973), A-179. Abstract \#701-54-39.

25. S. E. Warschawski, On the degree of variation in conformal mapping of variable regions, Trans. Amer. Math. Soc. 69 (1950), 335-356. MR 12, 327.

26. G. T. Whyburn, Analytic topology, Amer. Math. Soc. Colloq. Publ., vol. 28, Amer. Math. Soc., Providence, R. I., 1942. MR 4, 86.

\section{DEPARTMENT OF MATHEMATICS, UNIVERSITY OF TEXAS, EL PASO, TEXAS} 79968

Current address: 1706 Albans, Houston, Texas 77005 